\title{
Metformin-induced thyrotropin suppression is not associated with cardiac effects
}

\author{
Carlo Cappelli, ${ }^{1}$ Mario Rotondi, ${ }^{2}$ Ilenia Pirola, ${ }^{1}$ Barbara Agosti, ${ }^{3}$ Ana Maria Formenti, ${ }^{1}$ \\ Pasquale De Cata, ${ }^{2}$ Massimo Salvetti, ${ }^{1}$ Luca Chiovato, ${ }^{2}$ Maurizio Castellano ${ }^{1}$
}

\begin{abstract}
${ }^{1}$ Department of Medical and Surgical Sciences, Endocrine and Metabolic Unit, University of Brescia; ${ }^{2}$ Unit of Internal Medicine and Endocrinology, Fondazione Salvatore Maugeri Istituto di Ricovero e Cura a Carattere Scientifico, Istituto Superiore Prevenzione e Sicurezza Lavoro Laboratory for Endocrine Disruptors, University of Pavia; ${ }^{3}$ Diabetic Unit, Spedali Civili di Brescia; Italy
\end{abstract}

\begin{abstract}
OBJECTIVE: Metformin treatment may induce a decrease/suppression in serum TSH levels, mimicking sub-clinical hyperthyroidism (SHT). The aim of the present study was to retrospectively evaluate changes in several electrocardiographic indices in euthyroid subjects with diabetes who, after starting metformin treatment, developed a low serum TSH as compared to patients with SHT resulting from an underlying thyroid disease or TSH suppressive treatment with L-thyroxine. DESIGN: Heart rate, $P$ wave duration, $P$ wave dispersion, QTmax, QTmin and QT-dispersion were assessed in 23 patients with diabetes treated with metformin before and after 6 months of TSH-suppression and in 31 control patients with SHT. RESULTS: No significant changes in electrocardiographic parameters were observed from baseline to the TSH-suppression measurement. A significant difference in $P$ wave duration $(102.9 \pm 7.4$ vs. 92.1 $\pm 5.8 \mathrm{~ms}, \mathrm{p}<0.001)$, P wave dispersion $(13.1 \pm 3.4 \mathrm{vs} .7 .1 \pm 3.5 \mathrm{~ms}, \mathrm{p}<0.001)$, QTmax $(399 \pm 18$ vs. $388 \pm 16 \mathrm{~ms}, p=0.024)$, QTmin $(341 \pm 14$ vs. $350 \pm 17 \mathrm{~ms}, p=0.038)$ and $Q T$ dispersion $(49.9 \pm 9.6$ vs. $30.9 \pm 9.2 \mathrm{~ms}, \mathbf{p}<0.001)$ were observed between the control group with SHT and the group of diabetic patients with low serum levels of TSH. CONCLUSIONS: Our results show that the TSH-suppressive effect observed in patients taking metformin is not associated with peripheral markers of thyroid hormone excess, at least at the cardiac level.
\end{abstract}

Key words: Atrial fibrillation, Metformin, Sub-clinical hyperthyroidism

Address for correspondence:

Carlo Cappelli, MD,

Department of Medical and Surgical Sciences,

Endocrine and Metabolic Unit, University of Brescia c/o 1;

Medicina Spedali Civili di Brescia,

Piazzale Spedali Civili n ${ }^{\circ}$ 1, 25100 Brescia, Italy,

Tel.: ++390303995251, E-mail: cappelli@med.unibs.it

Received 08-04-2013, Accepted 16-09-2013

\section{INTRODUCTION}

Metformin is a widely used drug for the treatment of patients with type 2 diabetes mellitus, but also in insulin resistance syndromes. ${ }^{1-3}$ It is commonly regarded as a safe drug in that no clinically relevant pharmacologic interactions have been described when it is prescribed in combination with the other 
commonly used drugs, with the exception of folate and $\mathrm{B} 12 ., 5$

Recent data from the literature, including those from our groups, indicate that metformin influences serum levels of thyroid-stimulating hormone (TSH) by decreasing serum concentration of pituitary hormones both in hypothyroid patients treated with levothyroxine ${ }^{6,7}$ and in patients with diabetes with primary untreated hypothyroidism. ${ }^{8}$ Recently, a decrease of serum TSH was also observed in hypothyroid women being treated with metformin for an associated polycystic ovarian syndrome. ${ }^{9,10}$ This TSH-lowering effect of metformin has been extensively reported and, although the underlying mechanisms are not yet clear, several hypotheses have been addressed and well summarized in a recent review by Pappa and Alevizaki. ${ }^{11}$ However, the clinical significance of the low serum TSH remains yet to be clarified. Subclinical hyperthyroidism (SHT), characterized by low serum TSH and normal free thyroxine (fT4) and triiodothyronine (fT3) levels, may be due to either exogenous or endogenous causes. Although commonly asymptomatic, SHT is associated with an increased risk of cardiac arrhythmias, mostly atrial fibrillation, morphological and functional cardiac alterations, ${ }^{12,13}$ resulting in increased cardiovascular morbidity and mortality. ${ }^{14-16}$ It was also shown that patients with SHT suffer from a sympathetic-vagal imbalance, characterized by an increased sympathetic activity in the presence of a diminished vagal tone,,$^{12,13,17}$ and may also display a prolonged QT interval. ${ }^{18}$

The aim of the present study was to retrospectively evaluate the heart rate variability in euthyroid subjects with diabetes who, after starting metformin treatment, developed a low serum TSH. Their electrocardiographic parameters were compared with those observed in patients with SHT resulting from an underlying thyroid disease or TSH suppressive treatment with L-thyroxine. In particular, P wave duration (Pmax), P wave dispersion (PWD) and QT dispersion were measured. The cardiac evaluation was performed at baseline (i.e. before starting metformin treatment, when TSH was in the normal range) and at least 6 months after the development of a blunted serum TSH level. Our final goal was to clarify whether or not the metformin-induced reduction of serum TSH has electrocardiographic changes which, being similar to what is observed in real SHT, might warrant specific intervention.

\section{SUBJECTS AND METHODS}

Patients were recruited by searching the computerized database of patients followed at the Diabetic Unit of Department of Medical and Surgical Sciences, University of Brescia, Italy. Search criteria included the availability of: 1) a complete medical history; 2) a detailed drug treatment schedule including any change during the study period; 3 ) a full thyroid hormone profile. We excluded patients with diabetes in whom the low metformin-induced serum TSH level was observed for less than 6 months after initiation of treatment with metformin. Patients with cardiovascular illnesses, including hypertension, even if treated, with cardiovascular autonomic dysfunction and smokers were also excluded. None of the patients enrolled in the study was taking $\beta$-blockers, anti-arrhythmic drugs or anticholinergic drugs.

Among a total of 7020 patients with diabetes, 23 subjects (18 females and 5 males; mean age $54 \pm 7$ years), who developed a low serum TSH $(<0.4 \mathrm{mU} / \mathrm{L})$ after starting metformin treatment, satisfied the inclusion and exclusion criteria. Serum tests for TSH, fT4 and fT3 were available at baseline (i.e. before metformin treatment) and repeated every 6 months. The control group included 31 patients $(23$ females and 8 males; mean age $52.9 \pm 8.1$ years) with SHT who also satisfied the above described inclusion and exclusion criteria and had, at the time of evaluation, a low serum TSH lasting for at least 6 months. SHT was due either to a hyperfunctioning thyroid nodule $(\mathrm{n}=17)$ or to L-thyroxine TSH suppressive treatment for thyroid cancer $(n=14)$.

\section{Cardiovascular assessment}

Electrocardiography (ECG) was performed at $0.025(25 \mathrm{~mm} / \mathrm{sec})$ in the supine position. Patients were allowed to breathe normally during ECG. The P wave duration was measured manually: the starting point of the $\mathrm{P}$ wave was taken at the junction of the deflection of the $\mathrm{P}$ wave from the iso-electric line. The end point of the $\mathrm{P}$ wave was taken at the junction of the end of the $\mathrm{P}$ wave and the iso-electric line. The Pmax and the minimum $\mathrm{P}$ duration were measured on ECG recordings. The $\mathrm{P}$ wave dispersion was calcu- 
lated as a Pmax-minumum $\mathrm{P}$ duration. ${ }^{19} \mathrm{QT}$ intervals were measured manually in all the 12 leads from the onset of the QRS complex to the end of the T wave. Three consecutive QT intervals were measured and averaged for each lead. A minimum of nine leads in which the QT interval could be measured was required for QT dispersion to be determined. QT dispersion was defined as the difference between the longest and shortest QT interval. A skilled electrocardiography physician, blinded as to the patient's clinical status, reviewed all the electrocardiograms.

\section{Thyroid function tests}

Serum concentrations of fT4 (normal range: 8.0$19.0 \mathrm{pg} / \mathrm{mL}$, analytical sensitivity $1 \mathrm{pg} / \mathrm{mL}$; intra- and inter-assay coefficient of variation, $2.4 \%$ and $6.8 \%$, respectively), fT3 (normal range: 2.4-4.7; analytical sensitivity $0.35 \mathrm{pg} / \mathrm{mL}$; intra- and inter-assay coefficient of variation, $4.6 \%$ and $6.5 \%$, respectively), and $\mathrm{TSH}$ (normal range: $0.4-4.5 \mathrm{mIU} / \mathrm{L}$, analytical sensitivity $0.004 \mathrm{mlU} / \mathrm{L}$; intra- and inter-assay coefficient of variation, $2.5 \%$ and $5.7 \%$, respectively) were measured using immunochemiluminescent assays by an automated analyzer (Immulite 2000, DPC Cirrus, Los Angeles, CA, USA) employing commercial kits (Diagnostic Products Corporation, Los Angeles, CA, USA).

\section{Statistical analysis}

Statistical analysis was performed using SPSS software (SPSS, Inc., Evanston, IL). Longitudinal measures were compared by ANOVA or by the Wilcoxon test according to a normal or a nonparametric distribution of the tested variable. Between groups comparisons were performed by Student's t-test for unpaired data or by the Mann-Whitney $U$-test according to a normal or a nonparametric distribution of the tested variable. Correlation between two variables was ascertained by Pearson or Spearman's correlation tests, as appropriate. Frequencies among groups were compared by the $\chi^{2}$ test with Fisher's correction, when appropriate. A $p$-value $<0.05$ was considered statistically significant. Data are expressed as mean \pm standard deviation (sd), unless otherwise stated.

\section{RESULTS}

The clinical and biochemical features of the 23 patients with diabetes at baseline (i.e. before met- formin treatment) and at least 6 months after the development of a low serum TSH (i.e. after initiation of treatment with metformin) are summarized in Table 1. A significant improvement of glycated haemoglobin was observed during the investigation period $[7.3 \%(56 \mathrm{mmol} / \mathrm{mol}) \pm 0.9 \mathrm{vs} 6.8 \%(51 \mathrm{mmol} / \mathrm{mol})$ $\pm 0.6, \mathrm{p}<0.05]$, whereas a slight but non-significant reduction of body mass index (BMI) was found when data before and after metformin were compared $\left(27.4 \pm 0.9\right.$ vs $\left.26.9 \pm 1.1 \mathrm{Kg} / \mathrm{m}^{2}\right)$. No significant change was observed for the serum levels of fT4 and fT3. More importantly, no significant change in heart rate (76.3 $\pm 6.1 \mathrm{vs} 73.9 \pm 7 \mathrm{bpm})$, systolic (131.8 $\pm 8.4 \mathrm{vs}$ $129.3 \pm 4.6 \mathrm{mmHg})$ and diastolic $(83.4 \pm 9.3$ to $81.2 \pm 8.7$ $\mathrm{mmHg}$ ) blood pressure was observed.

Data regarding $\mathrm{P}$ wave dispersion and measurements of the QT interval are displayed in Table 2. When the results obtained at baseline and after at least 6 months of low serum TSH were compared, no significant change was observed in the $\mathrm{P}$ wave duration (91.4 \pm 6.9 vs $92.1 \pm 5.8 \mathrm{~ms})$, P wave dispersion (7.4 \pm 3.7 vs $7.1 \pm 3.5 \mathrm{~ms})$, QTmax $(390 \pm 17$ vs $388 \pm 16 \mathrm{~ms})$, QTmin $(348 \pm 18$ vs $350 \pm 17 \mathrm{~ms})$ and QT dispersion ( $30.8 \pm 9.1$ to $30.9 \pm 9.2 \mathrm{~ms}$ ).

To further evaluate this finding, patients with diabetes who developed a low serum TSH while treated with metformin were compared with control patients

Table 1. Clinical and biochemical features of patients with diabetes at baseline (i.e. before metformin treatment) and at least 6 months after the development of a low serum TSH

\begin{tabular}{lccc}
\hline & $\begin{array}{c}\text { Baseline, } \\
\text { before metformin } \\
\text { treatment }\end{array}$ & $\begin{array}{c}\text { After at least } \\
\text { 6 months of low } \\
\text { serum TSH }\end{array}$ & p value \\
\hline BMI $\left(\mathrm{Kg} / \mathrm{m}^{2}\right)$ & $27.4 \pm 0.9$ & $26.9 \pm 1.1$ & $\mathrm{NS}$ \\
$\begin{array}{l}\text { Heart Rate } \\
\text { (bpm) }\end{array}$ & $76.3 \pm 6.1$ & $73.9 \pm 7.0$ & $\mathrm{NS}$ \\
Systolic Blood & $131.8 \pm 8.4$ & $129.3 \pm 4.6$ & $\mathrm{NS}$ \\
$\begin{array}{l}\text { Pressure } \\
\text { (mmHb) }\end{array}$ & & & \\
Diastolic & $83.4 \pm 9.3$ & $81.2 \pm 8.7$ & $\mathrm{NS}$ \\
Heart Pressure & & & \\
$(\mathrm{mmHb})$ & $7.3 \pm 0.9$ & $6.8 \pm 0.6$ & $<0.05$ \\
HbA1c $(\%)$ & $1.1 \pm 0.7$ & $0.1 \pm 0.2$ & $<0.001$ \\
TSH $(\mathrm{mIU} / \mathrm{L})$ & $12.4 \pm 1.7$ & $12.6 \pm 1.4$ & $\mathrm{NS}$ \\
fT4 $(\mathrm{pg} / \mathrm{mL})$ & $2.9 \pm 0.6$ & $3.0 \pm 0.8$ & $\mathrm{NS}$ \\
fT3 $(\mathrm{pg} / \mathrm{mL})$ & & & \\
\hline
\end{tabular}


Table 2. Heart rate variability of patients with diabetes at baseline (i.e. before metformin treatment) and at least 6 months after the development of a low serum TSH

\begin{tabular}{lccc}
\hline & $\begin{array}{c}\text { Baseline, before } \\
\text { metformin } \\
\text { treatment }\end{array}$ & $\begin{array}{c}\text { After at least } \\
\text { 6 months of low } \\
\text { serum TSH }\end{array}$ & $\begin{array}{c}\mathbf{p} \\
\text { value }\end{array}$ \\
\hline Pmax (ms) & $91.4 \pm 6.9$ & $92.1 \pm 5.8$ & NS \\
PWD (ms) & $7.4 \pm 3.7$ & $7.1 \pm 3.5$ & NS \\
QTmax (ms) & $390 \pm 17$ & $388 \pm 16$ & NS \\
QTmin (ms) & $348 \pm 18$ & $350 \pm 17$ & NS \\
Qt dispersion (ms) & $30.8 \pm 9.1$ & $30.9 \pm 9.2$ & NS \\
\hline
\end{tabular}

having SHT. As shown in Table 3, the two groups displayed similar clinical and biochemical parameters. In particular, no difference was found for circulating thyroid function parameters, alcohol consumption, blood pressure and for heart rate. In addition, the mean period of low serum TSH was super-imposable between patients with diabetes and control group with SHT ( $1.0 \pm 0.7$ vs $1.1 \pm 0.8$ years, respectively). However, when the electrocardiographic measures were compared between the two groups, significant differences were observed. As shown in Figure 1, P wave duration (102.9 \pm 7.4 vs. $92.1 \pm 5.8 \mathrm{~ms}, \mathrm{p}<0.001)$,

Table 3. Clinical and biochemical features of patients with diabetes with TSH suppression and subjects with subclinical hyperthyroidism

\begin{tabular}{lccc}
\hline & $\begin{array}{c}\text { Diabetics with } \\
\text { low serum } \\
\text { TSH while } \\
\text { treated with } \\
\text { metformin (23) }\end{array}$ & $\begin{array}{c}\text { Control } \\
\text { patients } \\
\text { with SHT } \\
\mathbf{( 3 1 )}\end{array}$ & $\begin{array}{c}\text { p } \\
\text { value }\end{array}$ \\
\hline Age (years) & $54.7 \pm 9.8$ & $52.9 \pm 8.1$ & NS \\
BMI (Kg/m $\left.{ }^{2}\right)$ & $26.9 \pm 1.1$ & $27.5 \pm 0.5$ & NS \\
$\begin{array}{l}\text { Alcohol intake } \\
\text { (2/3 glasses of }\end{array}$ & $2 / 23$ & $3 / 31$ & NS \\
wine daily) (n) & $73.9 \pm 7.0$ & $80.3 \pm 4.1$ & NS \\
Heart Rate (bpm) & $133.7 \pm 4.4$ & NS \\
Systolic Blood & $129.3 \pm 4.6$ & & \\
Pressure (mmHb) & & $83.4 \pm 6.3$ & NS \\
Diastolic Blood & $81.2 \pm 8.7$ & & \\
Pressure (mmHb) & & $0.1 \pm 0.2$ & NS \\
TSH (mIU/L) & $0.1 \pm 0.2$ & $12.8 \pm 0.7$ & NS \\
fT4 (pg/mL) & $12.6 \pm 1.4$ & $3.1 \pm 0.6$ & NS \\
fT3 (pg/mL) & $3.0 \pm 0.8$ & & \\
\hline
\end{tabular}

P wave dispersion $(13.1 \pm 3.4$ vs. $7.1 \pm 3.5 \mathrm{~ms}, \mathrm{p}<0.001)$, QTmax (399 \pm 18 vs. $388 \pm 16 \mathrm{~ms}, \mathrm{p}=0.024)$, QTmin $(341 \pm 14$ vs. $350 \pm 17 \mathrm{~ms}, \mathrm{p}=0.038)$ and QT dispersion $(49.9 \pm 9.6$ vs. $30.9 \pm 9.2 \mathrm{~ms}, \mathrm{p}<0.001)$ were significantly different in subjects with SHT as compared to patients with diabetes having a low serum TSH while treated with metformin.

\section{DISCUSSION}

This study demonstrates that the blunted serum TSH induced by metformin treatment is not associated with changes of electrocardiographic parameters found in patients with real SHT. Indeed, significant differences were found when comparing Pmax, PWD, QTmax, QTmin and QT dispersion between patients with diabetes developing a low serum TSH while treated with metformin and patients with real SHT.

Since the first report by Vigersky $\mathrm{R}$ et $\mathrm{al}^{6}$ showing that metformin was able to decrease serum TSH levels in patients with diabetes and hypothyroidism, other studies confirmed this observation. ${ }^{6,8}$ In contrast, a recent analysis by Díez and Iglesias of a large cohort of patients with diabetes showed no independent and significant relationship between TSH values and metformin treatment in euthyroid patients with diabetes..$^{20}$ Unfortunately, no data about TSH serum levels before metformin treatment were given; it is thus possible that the TSH levels of patients on metformin could be higher before its introduction. Recently, we have elaborated on this including euthyroid patients with diabetes and borderline high serum TSH levels ( $>2.5 \mathrm{mU} / \mathrm{L}$ ) independently of the presence of thyroid antibodies. ${ }^{21}$

A major question regarding the metformin-induced reduction of serum TSH is whether or not this condition can be considered equivalent to SHT. As in fact suggested by Alevizaki, metformin could prove to be a very efficacious tool to induce TSH suppression without iatrogenic hyperthyroidism effects in thyroid cancer patients. ${ }^{22}$ Indeed, SHT is defined as a condition of lower than normal serum TSH associated with fT4 and fT3 in the normal range. ${ }^{23}$ SHT, which may result from endogenous thyroid diseases or exogenous factors, such as L-thyroxine treatment at TSH suppressive doses, is associated with corresponding alterations of the cardiovascular system, including 

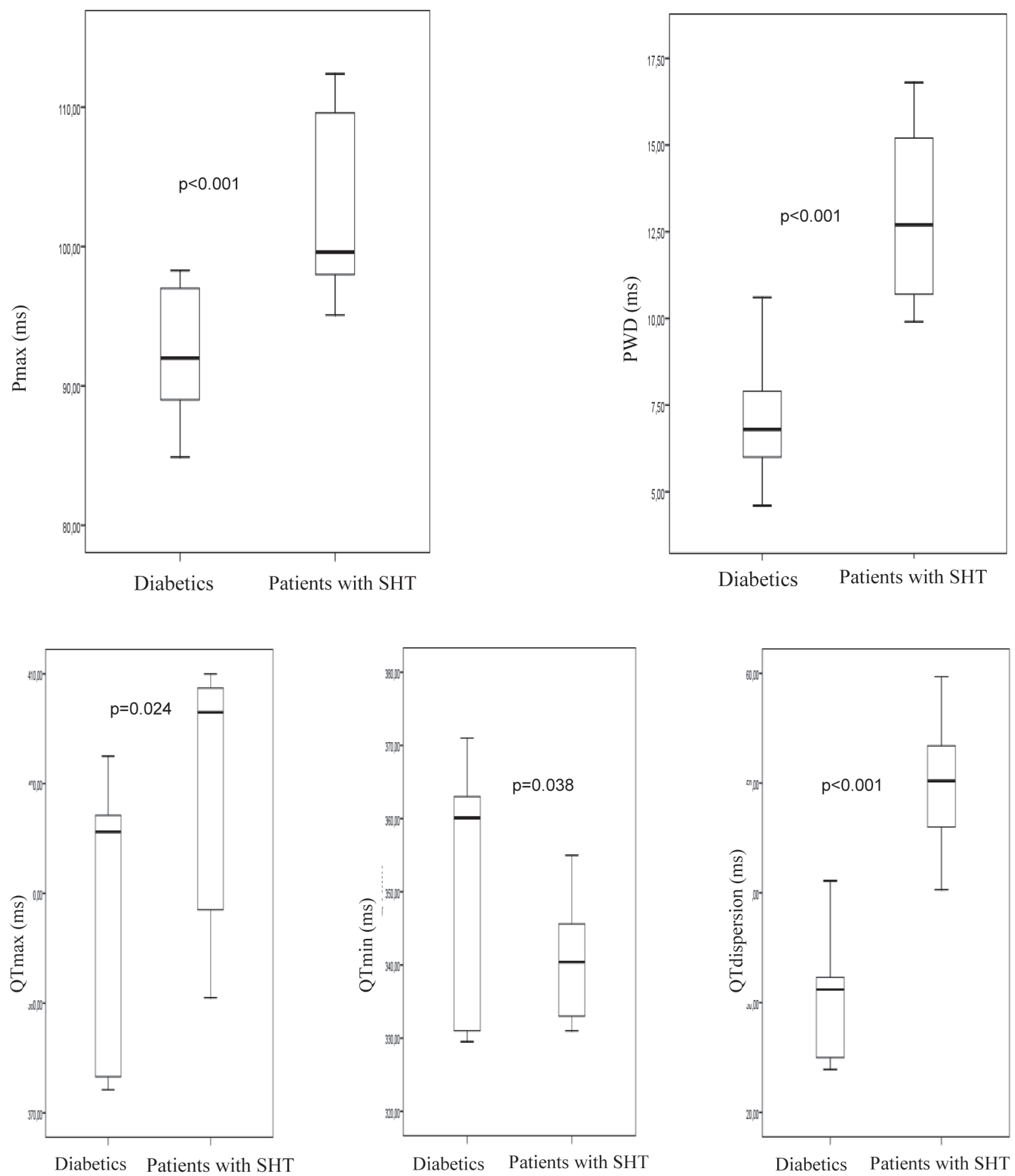

Figure 1. Heart rate variability of patients with diabetes with low serum TSH and patients affected by subclinical hyperthyroidism.

morphological and functional cardiac alterations, and an increased risk of cardiac arrhythmias (mostly atrial fibrillation) ${ }^{23-29} \mathrm{~A}$ recent meta-analysis, which included more than 52,000 subjects, clearly indicated that SHT is also associated with increased risk of coronary heart events and mortality. ${ }^{14}$ 
One of the earliest cardiovascular consequences of SHT is the occurrence of a sympathetic-vagal imbalance, characterized by increased sympathetic activity in the presence of a diminished vagal tone. ${ }^{12,13,17}$ Owecki et al also showed that, in its early stages, SHT causes alterations of ventricular repolarization, represented by a prolonged QT interval. ${ }^{18}$ These electrocardiographic alterations are apparently due to the condition of mild hyperthyroidism, as assessed by the lower than normal serum TSH, rather than to an aetiology of thyrotoxicosis. ${ }^{8,30}$

The present study did not show alterations in sympathetic activity in patients with diabetes who experienced a metformin-induced, prolonged reduction of serum TSH to lower than normal levels. Indeed, Pmax, PWD, QTmax, QTmin and QT did not change throughout the study duration in patients with diabetes. This was at variance with patients with real SHT, who displayed significantly increased measurements for these electrocardiographic parameters. The latter finding is in agreement with the recent literature. ${ }^{13,17}$

Taken together, our findings support the view that the reduction of serum TSH induced by metformin is not a manifestation of thyrotoxicosis but rather a "central" phenomenon involving a resetting of pituitary TSH secretion. This "effect" could have a practical clinical relevance. Indeed, TSH suppression could be achieved without the iatrogenic effects due to high doses of L-T4 normally used for this purpose, reducing the risk of development of cardiac hypertrophy (increase of left ventricular mass index), diastolic dysfunction and arrhythmias (mostly atrial fibrillation) ${ }^{23}$ osteoporosis/osteoporotic fracture, ${ }^{31}$ cognitive impairment $t^{32}$ and, moreover, of overall cause of mortality. ${ }^{33,34}$

Our interpretation is in line with the reported observation that the blunted TSH induced by metformin is not accompanied by clinical symptoms of hyperthyroidism ${ }^{6}$ or by any significant change of serum fT4 and fT3.

At the moment, the mechanisms by which metformin lowers serum TSH remain to be elucidated. As recently reviewed by Duntas, an emerging hypothesis to explain the TSH lowering effect of metformin involves the inhibition produced by this drug on hypothalamic adenosine 5'-monophosphate-activated protein kinase (AMPK). ${ }^{35,36}$ Although few studies on the regulation of the hypothalamic isoforms (a1 and a2) of AMPK are available, the results provided by Lopez et al would support the concept that the action of metformin on AMPK activity could counteract the effects of T3 at the hypothalamic level. ${ }^{37}$ The resulting effects on TSH secretion are still incompletely understood. As a consequence, further studies are needed to fully explain the TSH lowering effect of metformin.

The small number of enrolled patients with diabetes, the relatively short period (6 months) of metformin-induced TSH suppression and the retrospective nature of the present study could represent a few limitations. However, the duration of TSH suppression was similar in control patients with endogenous or exogenous SHT.

In conclusion, our results suggest that the low serum TSH observed in patients taking metformin is not associated with signs of thyroid hormone excess at the cardiac level. As a consequence, if these data are confirmed in large prospective studies, this drug-induced biochemical condition will potentially no longer be viewed as indicative of SHT. Thus, the clinical message would be that there is no need to adjust the L-thyroxine dose in hypothyroid patients started on metformin, nor is there a need for strict thyroid biochemical surveillance of patients with diabetes taking metformin.

\section{REFERENCES}

1. DeFronzo RA, Goodman AM, 1995 Efficacy of metformin in patients with non insulin-dependent diabetes mellitus. The Multicenter Metformin Study Group. N Eng J Med 333: 541.

2. UK Prospective Diabetes Study (UKPDS) Group, 1998 Effect of intensive blood-glucose control with metformin on complications in overweight patients with type 2 diabetes (UKPDS 34). Lancet 352: 854.

3. Abdelghaffar S, Attia A, 2009 Metformin added to insulin therapy for type 1 diabetes mellitus in adolescents. Coch Data Sys Rev 21: CD006691.

4. Scheen AJ, 2005 Drug interactions of clinical importance with antihyperglycaemic agents: an update. Drug Saf 28: 601-631.

5. Stocker DJ, Vigersky RA, Stocker DJ, 2005 The effects of metformin and rosiglitazone on vitamin B12, folate and homocysteine in patients with poorly controlled type 2 diabetes. Abstract book of the 87th Annual Meeting of 
The Endocrine Society; Abstract P3: 604-693.

6. Vigersky RA, Filmore-Nassar A, Glass AR, 2006 Thyreotropin suppression by metformin. J Clin Endocrinol Metab 91: 225-227.

7. Isidro ML, Penìn MA, Nemina R, Cordida F, 2007 Metformin reduces thyrotropin levels in obese, diabetic women with primary hypothyroidism on thyroidism on thyroxine replacement therapy. Endocrine 32: 79-82.

8. Cappelli C, Rotondi M, Pirola I, et al, 2009 TSH-lowering effect of metformin in type 2 patients with diabetes. Diabetes Care 32: 1589-1590.

9. Morteza Taghavi S, Rokni H, Fatemi S, 2011 Metformin decreases thyrotropin in overweight women with polycystic ovarian syndrome and hypothyroidism. Diab Vasc Dis Res 8: 47-48.

10. Rotondi M, Cappelli C, Magri F, et al, 2011 Thyroidal effect of metformin treatment in patients with polycystic ovary syndrome. Clin Endocrinol 75: 378-381.

11. Pappa T, Alevizaki M, 2013 Metformin and thyroid: an update. Eur Thyroid J 2: 22-28.

12. Portella RB, Pedrosa RC, Coeli CM, Buescu A, Vaisman M, 2007 Altered cardiovascular vagal responses in nonelderly female patients with subclinical hyperthyroidism and no apparent cardiovascular disease. Clin Endocrinol 67: 290-294.

13. Petretta M, Bonaduce D, Spinelli L, et al, 2001 Cardiovascular haemodynamics and cardiac autonomic control in patients with subclinical and overt hyperthyroidism. Eur J Endocrinol 145: 691-696.

14. Collet TH, Gussekloo J, Bauer DC, et al, 2012 Thyroid Studies Collaboration. Subclinical hyperthyroidism and the risk of coronary heart disease and mortality. Arch Intern Med 172: 799-809.

15. Kikuya M, Hozawa A, Ohokubo T, et al, 2000 Prognostic significance of blood pressure and heart rate variabilities: the Ohasama study. Hypertension 36: 901-906.

16. Shah S, Kambur T, Chan C, et al, 2013 Relation of short-term heart rate variability to incident heart failure (from the multi-Ethnic study of atherosclerosis). Am J Cardiol 112: 533-540.

17. Galetta F, Franzoni F, Fallahi P, et al, 2009 Changes in autonomic regulation and ventricular repolarization induced by subclinical hyperthyroidism. Biomed Pharmacoter 64: 546-549.

18. Owecki M, Michalak A, Nikisch E, Sowiński J, 2006 Prolonged ventricular repolarization measured by corrected QT interval (QTc) in subclinical hyperthyroidism. Horm Metab Res 38: 44-47.

19. Smit JW, Eustatia-Rutten CF, Corssmit EP, et al, 2005 Reversible diastolic dysfunction after long-term exogenous subclinical hyperthyroidism: a randomized, placebo-controlled study. J Clin Endocrinol Metab 90: 6041-6047.

20. Díez JJ, Iglesias P, 2013 Relationship between serum thyrotropin concentrations and metformin therapy in euthyroid patients with type 2 diabetes. Clin Endocrinol 78: 505-511.

21. Cappelli C, Rotondi M, Pirola I, et al, 2012 Thyreotropin levels in patients with diabetes on metformin treatment. Eur J Endocrinol 167: 261-265.

22. Alevizaki M, 2013 Metformin and thyroid: some questions still remain. Clin Endocrinol 78: 503-504.

23. Biondi B, Cooper DS, 2012 The clinical significance of subclinical thyroid dysfunction. Endocr Rev 29: 76-131.

24. Parle JV, Maisonneuve P, Sheppard MC, Boyle P, Franklyn JA, 2001 Prediction of all-cause and cardiovascular mortality in elderly people from one low serum thyrotropin result: a 10-year cohort study. Lancet 358: 861-865.

25. Iervasi G, Molinaro S, Landi P, et al, 2007 Association between increased mortality and mild thyroid dysfunction in cardiac patients. Arch Intern Med 167: 1526-1532.

26. Sawin CT, Geller A, Wolf PA, 1994 Low serum thyrotropin concentrations as a risk factor for atrial fibrillation in older persons. N Eng J Med 331: 1249-1252.

27. Cappola AR, Fried LP, Arnold AM, et al, 2006 Thyroid status, cardiovascular risk, and mortality in older adults. JAMA, 295: 1033-1041.

28. Auer J, Scheibner P, Mische T, Langsteger W, Eber O, Eber B, 2001 Subclinical hyperthyroidism as a risk factor for atrial fibrillation. Am Heart J 142: 838-842.

29. Cooper DS, Biondi B, 2012. Subclinical thyroid disease. Lancet 379: 1142-1154.

30. Gen R, Akbay E, Camsari A, Ozcan T, 2010 P-wave dispersion in endogenous and exogenous subclinical hyperthyroidism. J Endocrinol Invest 33: 88-91.

31. Vadiveloo T, Donnan PT, Cochrane L, Leese GP, 2011 The thyroid epidemiology, audit, and research study (TEARS) morbidity in patients with endogenous subclinical hyperthyroidism. J Clin Endocrinol Metab 96: 1344-1351.

32. Ceresini G, Lauretani F, Maggio M, et al, 2009 Thyroid function abnormalities and cognitive impairment in elderly people: results of the Invecchiare in Chianti study. J AM Geriatr Soc 57: 89-93.

33. Collet TH, Gussekloo J, Bauer DC, et al, 2012 Subclinical hyperthyroidism and the risk of coronary heart disease and mortality. Arch Intern Med 172: 799-809.

34. Sgarbi JA, Matsumurs LK, Kasamatsu, et al, 2010 Subclinical thyroid dysfunctions are independent risk factors for mortality in a 7.5-year follow-up: the JapaneseBrazilian thyroid study. Eur J Endocrinol 162: 569-577.

35. Duntas LH, Orgiazzi J, Brabant G, 2011 The interface between thyroid and diabetes mellitus. Clin Endocrinol 75: 1-9.

36. Lim CT, Kola B, Korbonits M, 2010 AMPK as a mediator of hormonal signalling. J Mol Endocrinol 44: 87-97.

37. Lòpez M, Varela L, Vàzquez MJ, et al, 2010 Hypothalamic AMPK and fatty acid metabolism mediate thyroid regulation of energy balance. Nat Med 16: 1001-1008. 